International Mathematical Forum, Vol. 9, 2014, no. 2, 51 - 63

HIKARI Ltd, www.m-hikari.com

http://dx.doi.org/10.12988/imf.2014.311215

\title{
On $p$-Compact Sets in Classical Banach Spaces
}

\author{
Juan Manuel Delgado \\ Departamento de Matemática Aplicada I \\ Escuela Técnia Superior de Arquitectura \\ Avenida Reina Mercedes, 2 \\ 41012 Seville, Spain \\ Cándido Piñeiro \\ Departamento de Matemáticas \\ Facultad de Ciencias Experimentales \\ Campus Universitario del Carmen \\ 21071 Huelva, Spain
}

Copyright (c) 2014 Juan Manuel Delgado and Cándido Piñeiro. This is an open access article distributed under the Creative Commons Attribution License, which permits unrestricted use, distribution, and reproduction in any medium, provided the original work is properly cited.

\begin{abstract}
Given $p \geq 1$, we denote by $\mathcal{C}_{p}$ the class of all Banach spaces $X$ satisfying the equality $\mathcal{K}_{p}(Y, X)=\Pi_{p}^{d}(Y, X)$ for every Banach space $Y$, $\mathcal{K}_{p}$ (respectively, $\Pi_{p}^{d}$ ) being the operator ideal of $p$-compact operators (respectively, of operators with $p$-summing adjoint). If $X$ belongs to $\mathcal{C}_{p}$, a bounded set $A \subset X$ is relatively $p$-compact if and only if the evaluation map $U_{A}^{*}: X^{*} \longrightarrow \ell_{\infty}(A)$ is $p$-summing. We obtain $p$-compactness criteria valid for Banach spaces in $\mathcal{C}_{p}$.
\end{abstract}

Mathematics Subject Classification: 46B50, 47B07, 47B10

Keywords: $p$-compact set, $p$-nuclear operator, $p$-summing operator, $(p, q)$ summing sequence 


\section{Introduction}

By a well known characterization due to Grothendieck [11], a subset $A$ of a Banach space $X$ is relatively compact if and only if there exists $\left(x_{n}\right)$ in $c_{0}(X)$ (the space of norm-null sequences in $X$ ) such that $A \subset\left\{\sum_{n} a_{n} x_{n}: \sum_{n}\left|a_{n}\right| \leq\right.$ $1\}$. Several authors have dealt with stronger forms of compactness studying sets sitting inside the convex hulls of special types of null sequences. For instance, it was observed in [20] (see also [5]) that if one considers, instead of $c_{0}(X)$, the space of $q$-summable sequences $\ell_{q}(X)$, for some fixed $q \geq 1$, then this stronger form of compactness characterizes the Reinov's approximation property of order $p, 0<p<1$. This latter form of compactness was recently further strengthened by Sinha and Karn [21] as follows. Let $1 \leq p \leq \infty$ and let $p^{\prime}$ be the conjugate index of $p$ (i.e., $1 / p+1 / p^{\prime}=1$ ). The $p$-convex hull of a sequence $\left(x_{n}\right) \in \ell_{p}(X)$ is defined as $p$-co $\left(x_{n}\right)=\left\{\sum_{n} a_{n} x_{n}: \sum_{n}\left|a_{n}\right|^{p^{\prime}} \leq 1\right\}$ $\left(\sup \left|a_{n}\right| \leq 1\right.$ if $\left.p=1\right)$. A set $A \subset X$ is said to be relatively $p$-compact if there exists $\left(x_{n}\right) \in \ell_{p}(X)\left(\left(x_{n}\right) \in c_{0}(X)\right.$ if $\left.p=\infty\right)$ such that $A \subset p$-co $\left(x_{n}\right)$. This nice notion has provoked the interest of several authors (see, for instance, [2], [6], [8] and [14]), whose contributions have made possible a deeper acknowledge of $p$-compactness in arbitrary Banach spaces. Anyway, there is no much information or examples of relative $p$-compact sets in concrete Banach spaces.

In [8], it is proved that a bounded subset $A$ of an arbitrary Banach space $X$ is relatively $p$-compact if and only if the corresponding evaluation map $U_{A}^{*}: x^{*} \in X^{*} \longmapsto\left(\left\langle x^{*}, a\right\rangle\right)_{a \in A} \in \ell_{\infty}(A)$ is $p$-nuclear ([8, Proposition 3.5]). However, for a wide class, say $\mathcal{C}_{p}$, of Banach spaces, the relatively $p$-compactness of any bounded set $A$ occurs whenever $U_{A}^{*}$ is just $p$-summing. For instance, reflexive spaces or separable dual spaces belong to $\mathcal{C}_{p}$ for all $p \geq 1$. In Section 2, a characterization of relatively $p$-compact sets in Banach spaces belonging to $\mathcal{C}_{p}$ is given; as an application, we obtain a characterization of $p$-compact sets in $\ell_{1}$. Section 3 is devoted mainly to show some ways to produce relatively $p$-compact sets in Banach spaces not belonging to $\mathcal{C}_{p}$.

A Banach space $X$ will be regarded as a subspace of its bidual $X^{* *}$ under the canonical embedding $i_{X}: X \rightarrow X^{* *}$. We denote the closed unit ball of $X$ by $B_{X}$. For Banach spaces $X$ and $Y$, the Banach space of all bounded linear operators from $X$ to $Y$ is denoted by $\mathcal{L}(X, Y)$. If $\mathcal{A}$ is a Banach ideal, then $\mathcal{A}^{d}$ denotes its dual ideal, that is, $\mathcal{A}^{d}(X, Y)=\left\{T \in \mathcal{L}(X, Y): T^{*} \in\right.$ $\left.\mathcal{A}\left(Y^{*}, X^{*}\right)\right\}$. We deal with the following operator ideals: $\mathcal{N}_{p}-p$-nuclear operators, $Q \mathcal{N}_{p}-$ quasi $p$-nuclear operators, $\mathcal{J}_{p}-p$-integral operators and $\Pi_{p}-$ $p$-summing operators. We refer to Pietsch's book [18] for operator ideals (see also [9] by Diestel, Jarchow, and Tonge for common operator ideals as $\mathcal{N}_{p}$ and $\Pi_{p}$, and [17] by Persson and Pietsch for $Q \mathcal{N}_{p}$ ).

As usual, the space of all weakly $p$-summable sequences (respectively, $p$ - 
summable sequences) in $X$ is denoted by $\ell_{p}^{w}(X)$ (respectively, $\left.\ell_{p}(X)\right)$ endowed with its norm

$$
\begin{gathered}
\left\|\left(x_{n}\right)\right\|_{p}^{w}=\sup _{x^{*} \in B_{X^{*}}}\left(\sum_{n}\left|\left\langle x^{*}, x_{n}\right\rangle\right|^{p}\right)^{1 / p} . \\
\left(\text { respectively, }\left\|\left(x_{n}\right)\right\|_{p}=\left(\sum_{n}\left\|x_{n}\right\|^{p}\right)^{1 / p}\right) .
\end{gathered}
$$

Relying on the notion of $p$-compactness, the notion of $p$-compact operator is defined in an obvious way (see [21]): an operator $T \in \mathcal{L}(X, Y)$ is said to be $p$-compact if $T\left(B_{X}\right)$ is relatively $p$-compact in $Y$. The space of all $p$-compact operators from $X$ into $Y$ is denoted by $\mathcal{K}_{p}(X, Y)$. It is shown in [21] that $\mathcal{K}_{p}$ is an operator ideal. We list some properties related to $p$-compactness:

- If $1 \leq q \leq p \leq \infty$, every relatively $q$-compact set is relatively $p$ compact.

- An operator $T$ belongs to $\mathcal{K}_{p}(X, Y)$ (respectively, $\mathcal{Q N}_{p}(X, Y)$ ) if and only $T^{*}$ belongs to $\mathcal{Q} \mathcal{N}_{p}\left(Y^{*}, X^{*}\right)$ (respectively, $\mathcal{K}_{p}\left(Y^{*}, X^{*}\right)$ ) [8, Corollary 3.4 and Proposition 3.8].

\section{2 -Compactness and $p$-summing evaluation maps}

A bounded subset $A$ of a Banach space $X$ is relatively $p$-compact if and only if the corresponding evaluation map $U_{A}^{*}: x^{*} \in X^{*} \longmapsto\left(\left\langle x^{*}, a\right\rangle\right)_{a \in A} \in \ell_{\infty}(A)$ is (quasi) p-nuclear [8, Proposition 3.5]. Nevertheless, for a wide class of Banach spaces, the relative $p$-compactness of a set is characterized just by the $p$-summability of its evaluation map. For the time being, let us focus our attention on this type of spaces.

Definition 2.1. Let $1 \leq p<\infty$. A Banach space $X$ belongs to the class $\mathrm{C}_{p}$ if for every bounded subset $A$ of $X, A$ is relatively $p$-compact if and only if the evaluation map $U_{A}^{*}: x^{*} \in X^{*} \longmapsto\left(\left\langle x^{*}, a\right\rangle\right)_{a \in A} \in \ell_{\infty}(A)$ is $p$-summing.

Recall that $\mathcal{K}_{p}(Y, X) \subset \Pi_{p}^{d}(Y, X)$ [21, Proposition 5.3]. Related to this, the following are reformulations of the definition of the class $\mathcal{C}_{p}$.

Proposition 2.1. Let $1 \leq p<\infty$. The following statements are equivalent for a Banach space $X$ :

a) $X \in \mathcal{C}_{p}$.

b) $\mathcal{K}_{p}(Y, X)=\Pi_{p}^{d}(Y, X)$ for every Banach space $Y$. 
c) $\mathcal{K}_{p}\left(\ell_{1}(\Gamma), X\right)=\Pi_{p}^{d}\left(\ell_{1}(\Gamma), X\right)$ for any set $\Gamma$.

d) $\mathcal{K}_{p}\left(\ell_{1}, X\right)=\Pi_{p}^{d}\left(\ell_{1}, X\right)$.

Proof. a $\Rightarrow$ b) For a given Banach space $Y$, consider $T \in \Pi_{p}^{d}(Y, X)$ and put $A:=T\left(B_{Y}\right)$. Since $\left\|U_{A}^{*} x^{*}\right\|_{\infty}=\left\|T^{*} x^{*}\right\|$, we have that $U_{A}^{*}$ is $p$-summing so, by hypothesis, $A=T\left(B_{Y}\right)$ is relatively $p$-compact.

b) $\Rightarrow$ c) and c) $\Rightarrow$ d) are obvious.

d) $\Rightarrow$ a) Suppose $A \subset X$ is a bounded set such that $U_{A}^{*}$ is $p$-summing. To see that $A$ is relatively $p$-compact, it suffices to show that each countably subset of $A$ is relatively $p$-compact. So consider $\left\{x_{n}\right\} \subset A$ and define $J:\left(\alpha_{n}\right) \in \ell_{1} \longmapsto J\left(\alpha_{n}\right) \in \ell_{1}(A)$, where $J\left(\alpha_{n}\right)(x)=\alpha_{n}$ if $x=x_{n}$ and $J\left(\alpha_{n}\right)(x)=0$ otherwise. From d), it follows that $U_{A} \circ J: \ell_{1} \longrightarrow X$ is $p$ compact. Thus, $\left\{x_{n}\right\}=\left\{U_{A} \circ J\left(e_{n}\right)\right\}$ is relatively $p$-compact.

Remark 2.2. Since $\ell_{\infty}(\Gamma)$ is an injective space, $\Pi_{p}^{d}$ may be replaced with $\mathrm{J}_{p}^{d}$ in c) and $\mathrm{d}$ ) of the above proposition ([9, Corollary 5.7]). In the same direction, $\mathcal{K}_{p}$ may be replaced with $\mathcal{N}_{p}^{d}$ in the mentioned statements since $\mathcal{K}_{p}\left(\ell_{1}(\Gamma), X\right)=\mathcal{N}_{p}^{d}\left(\ell_{1}(\Gamma), X\right)$ for every Banach space $X$ ([8, Proposition 3.8] and [17, Theorem 38]). In particular, we have that $X$ belongs to $\mathcal{C}_{p}$ if and only if $\mathcal{N}_{p}^{d}\left(\ell_{1}, X\right)=\mathcal{J}_{p}^{d}\left(\ell_{1}, X\right)$.

The preceding remark reveals that the equality $\mathcal{N}_{p}(Y, Z)=\mathcal{J}_{p}(Y, Z)$ becomes of great use to provide examples of Banach spaces belonging to $\mathcal{C}_{p}$.

Proposition 2.2. Let $X$ be a Banach space and $1 \leq p<\infty$. Then

1. If $X^{* *}$ has the Radon-Nikodym property then $X \in \mathrm{e}_{p}$. In particular, every reflexive Banach space belongs to $\mathcal{C}_{p}$.

2. If $X^{* *} \in \mathcal{C}_{p}$ then $X \in \mathcal{C}_{p}$.

3. $c_{0}, \ell_{\infty} \notin \mathrm{C}_{p}$.

4. If $\mu$ is a finite measure, then $L_{1}(\mu) \notin \mathcal{C}_{p}$.

Proof. According to $\left[1\right.$, Proposition 1.1], we have that $\mathcal{N}_{p}\left(X^{*}, \ell_{\infty}(A)\right)=$ $\mathcal{J}_{p}\left(X^{*}, \ell_{\infty}(A)\right)$ whenever $X^{* *}$ has the Radon-Nykodim property.

To see 2 , consider $A \subset X$ such that $U_{A}^{*} \in \Pi_{p}\left(X^{*}, \ell_{\infty}(A)\right)$, that is,

$$
\left(\sum_{n=1}^{N}\left|\left\langle x_{n}^{*}, x_{n}\right\rangle\right|^{p}\right)^{1 / p} \leq \pi_{p}\left(U_{A}^{*}\right) \sup _{x \in B_{X}}\left(\sum_{n=1}^{N}\left|\left\langle x_{n}^{*}, x\right\rangle\right|^{p}\right)^{1 / p}
$$

for all finite subsets $\left\{x_{1}, \ldots, x_{N}\right\}$ in $A$ and $\left\{x_{1}^{*}, \ldots, x_{N}^{*}\right\}$ in $X^{*}$. It suffices to show that $i_{X}(A)$ is relatively $p$-compact in $X^{* *}([8$, Corollary 3.6]). Given 
finite subsets $\left\{x_{1}, \ldots, x_{N}\right\}$ in $A$ and $\left\{x_{1}^{* * *}, \ldots, x_{N}^{* * *}\right\}$ in $X^{* * *}$, we have from (1)

$$
\begin{aligned}
\left(\sum_{n=1}^{N}\left|\left\langle x_{n}^{* * *}, i_{X}\left(x_{n}\right)\right\rangle\right|^{p}\right)^{1 / p} & =\left(\sum_{n=1}^{N}\left|\left\langle i_{X}^{*}\left(x_{n}^{* * *}\right), x_{n}\right\rangle\right|^{p}\right)^{1 / p} \\
& \leq \pi_{p}\left(U_{A}^{*}\right) \sup _{x \in B_{X}}\left(\sum_{n=1}^{N}\left|\left\langle i_{X}^{*}\left(x_{n}^{* * *}\right), x\right\rangle\right|^{p}\right)^{1 / p} \\
& \leq \pi_{p}\left(U_{A}^{*}\right) \sup _{x^{* *} \in B_{X}^{* *}}\left(\sum_{n=1}^{N}\left|\left\langle x_{n}^{* * *}, x^{* *}\right\rangle\right|^{p}\right)^{1 / p}
\end{aligned}
$$

It follows from the above reasoning that the evaluation map of $i_{X}(A)$ is $p$ summing and, by hypothesis, $i_{X}(A)$ is relatively $p$-compact in $X^{* *}$.

Grothendieck's Theorem ensures that the natural embedding $i: \ell_{1} \longrightarrow c_{0}$ has $p$-summing adjoint since $i^{*}$ factors through $\ell_{2}$. So, if $c_{0} \in \mathcal{C}_{p}$ then $i \in$ $\mathcal{K}_{p}\left(\ell_{1}, c_{0}\right)$ (Proposition 2.1) which is a contradiction because $i$ is not even compact. Finally, 2 guarantees that $\ell_{\infty}$ does not belong to $\mathcal{C}_{p}$.

Finally, the formal identity $i_{1}: L_{\infty}(\mu) \longrightarrow L_{1}(\mu)$ is 1-integral, so $i_{1}^{*}$ is [9, Theorem 5.15]. Then, $i_{1}$ is $p$-summing for all $p \geq 1$. Nevertheless, $i_{1}$ is not $p$-compact for any $p \geq 1$ (in fact, it is not even compact). In view of Proposition 2.1b, $L_{1}(\mu) \notin \mathrm{C}_{p}$.

By definition, a 2-compact set $A$ in $X=\ell_{2}$ is that for which there exists a 2 -summable sequence $\left(x_{n}\right)$ in $X$ such that $A \subset\left\{\sum_{n} \alpha_{n} x_{n}:\left(\alpha_{n}\right) \in B_{\ell_{2}}\right\}$. The sequence $\left(x_{n}\right)$ yields the Hilbert-Schmidt operator $\phi: e_{n} \in \ell_{2} \longmapsto x_{n} \in X$ and we have $A \subset \phi\left(B_{\ell_{2}}\right)$. This idea establishes a way to obtain $p$-compact sets $(1 \leq p \leq 2)$ in Hilbert spaces:

Corollary 2.3. Let $X$ be a Hilbert space and $1 \leq p \leq 2$. A subset $A$ of $X$ is relatively $p$-compact if and only if there exists a Hilbert-Schmidt operator $\phi: \ell_{2} \longrightarrow X$ such that $A \subset \phi\left(B_{\ell_{2}}\right)$.

Proof. Since $X^{*}$ has cotype 2, it suffices to deal with $p=2$ ([19, Proposition 3.6]). Suppose $A \subset X$ is such that $A \subset \phi\left(B_{\ell_{2}}\right)$ for a given HilbertSchmidt operator $\phi: \ell_{2} \longrightarrow X$. Now, $\phi^{*} \in \Pi_{2}\left(X^{*}, \ell_{2}\right)$ [9, Theorem 4.10] and, by Proposition 2.1, $\phi \in \mathcal{K}_{2}\left(\ell_{2}, X\right)$. So $A \subset \phi\left(B_{\ell_{2}}\right)$ must be relatively 2-compact.

In order to show that $\ell_{1}(\Gamma) \in \mathcal{C}_{p}$ for any set $\Gamma$, we need the following

Lemma 2.4. Let $Y$ and $Z$ be Banach spaces. If $T: Y \longrightarrow Z^{*}$ is a weakly compact operator and $R:=T_{\left.\right|_{Z}}^{*}$, then $R^{* *}=T^{*}$. 
Proof. Let $z_{0}^{* *} \in B_{Z^{* *}}$ and choose a net $\left(z_{\delta}\right)_{\delta}$ in $B_{Z}$ such that

$$
z_{0}^{* *}=\sigma\left(Z^{* *}, Z^{*}\right)-\lim _{\delta} z_{\delta}
$$

Since $T^{*}$ is $\sigma\left(Z^{* *}, Z^{*}\right)-\sigma\left(Y^{*}, Y^{* *}\right)$-continuous, we have

$$
T^{*} z_{0}^{* *}=\sigma\left(Y^{*}, Y^{* *}\right)-\lim _{\delta} T^{*} z_{\delta}=\sigma\left(Y^{*}, Y^{* *}\right)-\lim _{\delta} R z_{\delta}
$$

On the other hand, since $R=T_{\left.\right|_{Z}}^{*}$ is also a weakly compact operator, it follows that $R^{* *}\left(Z^{* *}\right) \subset Y^{*}$ and $R^{* *}$ is $\sigma\left(Z^{* *}, Z^{*}\right)-\sigma\left(Y^{*}, Y^{* *}\right)$-continuous. Hence

$$
R^{* *} z_{0}^{* *}=\sigma\left(Y^{*}, Y^{* *}\right)-\lim _{\delta} R^{* *} z_{\delta}=\sigma\left(Y^{*}, Y^{* *}\right)-\lim _{\delta} R z_{\delta} T^{*} z_{0}^{* *}
$$

Corollary 2.5. Every separable dual space belongs to $\mathfrak{C}_{p}$.

Proof. Let $X=Z^{*}$ be a separable Banach space. It suffices to show that $\mathrm{J}_{p}^{d}\left(\ell_{1}, X\right) \subset \mathcal{N}_{p}^{d}\left(\ell_{1}, X\right)$ (Remark 2.2). Consider $T: \ell_{1} \longrightarrow X$ such that $T^{*} \in$ $\mathrm{J}_{p}\left(X^{*}, \ell_{\infty}\right)$. Now, $R=T_{\mid z}^{*}$ is also $p$-integral and, according to [16, Theorem 5], $p$-nuclear. From this and Lemma 2.4, we have $R^{* *}=T^{*}$ is $p$-nuclear.

Arguing as in the proof of $\mathrm{d}) \Rightarrow \mathrm{a}$ ) in Proposition 2.1, Corollary 2.5 yields Corollary 2.6. $\ell_{1}(\Gamma) \in \mathcal{C}_{p}$ for any set $\Gamma$.

Now, we deal with the problem of characterizing relatively $p$-compact sets in $\ell_{1}$. A necessary condition for a bounded subset $A \subset \ell_{1}$ to be relatively $p$-compact is that $U_{A}^{*}$ maps the weakly $p$-summable sequence $\left(e_{k}\right)$ in $\ell_{\infty}$ to a $p$-summable sequence in $\ell_{\infty}(A)$. In this case, given $a=(a(k)) \in A$ we have

$$
|a(k)|=\left|\left\langle a, e_{k}\right\rangle\right| \leq \sup _{a \in A}\left|\left\langle a, e_{k}\right\rangle\right|=\left\|U_{A}^{*} e_{k}\right\| .
$$

In other words, if $A \subset \ell_{1}$ is relatively $p$-compact then there exists $\gamma=$ $(\gamma(k)) \in \ell_{p}$ such that $|a(k)| \leq \gamma(k)$ for all $k \in \mathbb{N}$ and $a \in A$. Of course, the converse is not true when $p>1$ : if $a_{n}=(1 / n, . . ., 1 / n, 0, \ldots)$, the sequence $\left(a_{n}\right)$ is "dominated" by $\gamma=(1 / k)$ but it is not even relatively compact.

Corollary 2.7. A bounded subset $A \subset \ell_{1}$ is relatively 1-compact if and only if it is order bounded.

Proof. Suppose that $A \subset \ell_{1}$ is order bounded. In view of [9, Theorem 5.19], $U_{A}$ is 1-integral, so $U_{A}^{*}$ is. In particular, $U_{A}^{*}$ is 1 -summing and, according to Corollary 2.6, $A$ is relatively 1-compact. 
The criterion of $p$-compactness in $\ell_{1}(p>1)$ will need the following result that characterizes bounded sets with $p$-summing evaluation map. Recall that a sequence $\left(x_{n}\right)$ in $X$ is strongly $p$-summable if $\sum_{n}\left|\left\langle x_{n}^{*}, x_{n}\right\rangle\right|<\infty$ for all $\left(x_{n}^{*}\right) \in \ell_{p^{\prime}}^{w}\left(X^{*}\right)([7])$. This notion has been extended and studied later by several authors in a natural way: $\left(x_{n}\right) \subset X$ is said to be $(p, q)$-summing if $\sum_{n}\left|\left\langle x_{n}^{*}, x_{n}\right\rangle\right|^{p}<\infty$ for all $\left(x_{n}^{*}\right) \in \ell_{q}^{w}\left(X^{*}\right)$ (see, for instance, [3], [4] and [12]).

Theorem 2.8. Let $X$ be a Banach space and $p \geq 1$. The following statements are equivalent for a bounded set $A \subset X$ :

a) The evaluation map $U_{A}^{*}: X^{*} \longrightarrow \ell_{\infty}(A)$ is $p$-summing.

b) For all $\left(x_{n}\right) \in A^{\mathbb{N}}$ and $\beta=\left(\beta_{n}\right) \in \ell_{p^{\prime}}\left(\beta \in c_{0}\right.$ if $\left.p=1\right)$, the operator $\phi: \ell_{p} \longrightarrow X$ defined by $\phi\left(e_{n}\right)=\beta_{n} x_{n}$ is nuclear.

c) For all $\left(x_{n}\right) \in A^{\mathbb{N}}$ and $\beta=\left(\beta_{n}\right) \in \ell_{p^{\prime}}\left(\beta \in c_{0}\right.$ if $\left.p=1\right)$, the sequence $\left(\beta_{n} x_{n}\right)$ is strongly $p^{\prime}$-summable.

d) For all $\left(x_{n}\right) \in A^{\mathbb{N}}$, the sequence $\left(x_{n}\right)$ is $(p, p)$-summing.

Proof. a $\Rightarrow$ b) Fixed $\left(x_{n}\right) \in A^{\mathbb{N}}$ and $\beta=\left(\beta_{n}\right) \in \ell_{p^{\prime}}$, consider the operators

$$
\begin{aligned}
& D_{\beta}: \quad \ell_{p} \longrightarrow \ell_{1} \quad P: \ell_{\infty}(A) \longrightarrow \ell_{\infty} \\
& \left(\alpha_{n}\right) \longmapsto\left(\beta_{n} \alpha_{n}\right) \quad \xi \quad \xi\left(\xi\left(x_{n}\right)\right)
\end{aligned}
$$

The adjoint of $\phi$ factors as follows:

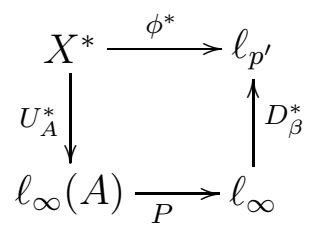

It is easy to check that $D_{\beta}^{*}=\sum_{n} \beta_{n} e_{n}^{*} \otimes e_{n}$ where $\left(e_{n}\right)$ and $\left(e_{n}^{*}\right)$ denote the unit vector basis of $\ell_{p^{\prime}}$ and $\ell_{1}$, respectively. Thus, $D_{\beta}$ is $p^{\prime}$-nuclear and, since $U_{A}^{*}$ is $p$-summing, we conclude that $\phi^{*}=D_{\beta}^{*} \circ P \circ U_{A}^{*} \in \mathcal{N}_{1}\left(X^{*}, \ell_{p^{\prime}}\right)([17$, Theorem 48]). According to [10, Theorem VIII.3.7], $\phi$ is a nuclear operator.

$\mathrm{b}) \Rightarrow \mathrm{c})$ According to $\left[3\right.$, Theorem 2], the space $\mathcal{J}_{1}\left(\ell_{p}, X\right)$ is isometrically isomorphic to the space of all strongly $p^{\prime}$-summable sequences in $X$ and the isometry is given by $\phi \in \mathcal{J}_{1}\left(\ell_{p}, X\right) \longmapsto\left(\phi e_{n}\right)$. Now, c) is concluded since every nuclear operator is, in particular, integral.

c) $\Rightarrow$ d) It is straightforward.

d) $\Rightarrow$ a) By contradiction, suppose $U_{A}^{*}$ is not $p$-summing. Then, for each $k \in \mathbb{N}$ there exist sequences $\left(x_{n, k}\right)_{n} \in A^{\mathbb{N}}$ and $\left(x_{n, k}^{*}\right)_{n} \in B_{\ell_{p}^{w}\left(X^{*}\right)}$ such that $\sum_{n}\left|\left\langle x_{n, k}^{*}, x_{n, k}\right\rangle\right|^{p} \geq k^{2 p}$. If $x \in X$,

$$
\sum_{k} \sum_{n}\left|\left\langle\frac{1}{k^{2}} x_{n, k}^{*}, x\right\rangle\right|^{p} \leq \sum_{k} \frac{1}{k^{2 p}},
$$


that is to say, $\left(k^{-2} x_{n, k}^{*}\right)_{n, k}$ is weakly $p$-summable in $X^{*}$. Nevertheless,

$$
\sum_{k} \sum_{n}\left|\left\langle\frac{1}{k^{2}} x_{n, k}^{*}, x_{n, k}\right\rangle\right|^{p} \geq \sum_{k} \frac{1}{k^{2 p}} k^{2 p}=\infty
$$

in contradiction to d).

Given a nuclear operator $\phi: \ell_{p} \longrightarrow \ell_{1}$, let us denote $\left(\sigma_{n}(k)\right)_{k}=\phi\left(e_{n}\right)$. Then $\phi^{*}$ is also nuclear and, in particular, 1-summing. Hence,

$$
\infty>\sum_{k}\left\|\phi^{*}\left(e_{k}^{*}\right)\right\|_{p^{\prime}}=\sum_{k}\left(\sum_{n}\left|\sigma_{n}(k)\right|^{p^{\prime}}\right)^{1 / p^{\prime}}
$$

where $\left(e_{k}\right)^{*}$ denotes the canonical vector sequence in $\ell_{\infty}$. Conversely, if the matrix $\left(\sigma_{n}(k)\right)_{n, k}$ verifies (2), then $\phi$ admits the nuclear representation $\sum_{n}\left(\sigma_{n}(k)\right)_{k} \otimes e_{k}$.

Corollary 2.9. Let $p>1$. A bounded subset $A \subset \ell_{1}$ is relatively $p$-compact if and only if

$$
\sum_{k}\left(\sum_{n}\left|\beta_{n} x_{n}(k)\right|^{p^{\prime}}\right)^{1 / p^{\prime}}<\infty
$$

for all $\left(x_{n}\right) \in A^{\mathbb{N}}$ and $\beta=\left(\beta_{n}\right) \in \ell_{p^{\prime}}$.

\section{Final notes}

In Proposition 2.2, we have mentioned that neither $c_{0}$ nor $\ell_{\infty}$ belong to $\mathcal{C}_{p}$. Anyway, we have the following way to generate 2-compact sets in $c_{0}$ : if $A \subset \ell_{2}$ is relatively compact, then $A$ is relatively 2 -compact as a subset of $c_{0}$. In fact, the identity map from $\ell_{2}$ to $c_{0}$ has 1-summing (hence, 2-summing) adjoint, so that operator maps relatively compact sets in $\ell_{2}$ to relatively 2 -compact sets in $c_{0}$ [8, Theorem 3.14]. This example inspires the following lemma:

Lemma 3.1. Let $X$ be a $\mathcal{L}_{\infty}$-space and $1 \leq p \leq 2$. Then $A \subset X$ is relatively $p$-compact if and only if there exist a relatively compact set $K \subset \ell_{2}$ and an operator $\phi: \ell_{2} \longrightarrow X$ such that $A \subset \phi(K)$.

Proof. The dual space $X^{*}$ is a $\mathcal{L}_{1}$-space. Hence, $X^{*}$ has cotype 2 , so it suffices to deal with $p=2$ ([19, Proposition 3.6]). If $A \subset X$ is relatively 2-compact, there exists $\left(x_{n}\right) \in \ell_{2}(X)$ such that $A \subset 2$-co $\left(x_{n}\right)$. Choose $\left(\alpha_{n}\right) \searrow 0$ so that $\left(\alpha_{n}^{-1} x_{n}\right)$ remains to be 2 -summable. Now consider the operators $D:\left(e_{n}\right) \in$ $\ell_{2} \longmapsto\left(\alpha_{n} e_{n}\right) \in \ell_{2}$ and $\phi: e_{n} \in \ell_{2} \longmapsto\left(\alpha_{n}^{-1} x_{n}\right) \in X$. It is clear that $A \subset \phi(K), K$ being the relatively compact set $D\left(B_{\ell_{2}}\right)$. Conversely, suppose $A \subset X$ is such that there exist a relatively compact set $K \subset \ell_{2}$ and an 
operator $\phi: \ell_{2} \longrightarrow X$ verifying $A \subset \phi(K)$. According to [9, Theorem 3.1], $\phi^{*}$ is 2 -summing, so $\phi$ map relatively compact sets in $\ell_{2}$ to relatively 2 -compact sets in $X$ [8, Theorem 3.14].

Given an absolutely convex and weakly compact set $B \subset X, \operatorname{span}(B)$ is denoted by $X_{B}$. This space is normed by the Minkowski's functional of $B$ :

$$
\rho_{B}(x)=\inf \{t>0: x \in t B\} .
$$

It is well known that $\left(X_{B}, \rho_{B}\right)$ is complete and $B$ is its closed unit ball. The canonical inclusion map from $X_{B}$ into $X$ is denoted by $j_{B}$.

Proposition 3.1. Let $X$ be a $\mathcal{L}_{\infty}$-space and $1 \leq p \leq 2$. Then $A \subset X$ is relatively $p$-compact if and only there exists $\left(x_{n}\right) \in \ell_{2}^{w}(X)$ such that the following conditions are satisfied:

1. $A \subset B:=2-\operatorname{co}\left(x_{n}\right)$;

2. $A$ is relatively compact in $X_{B}$.

Proof. As in the previous proof, it suffices to deal with the case $p=2$. If $A \subset X$ is relatively 2-compact, Lemma 3.1 guarantees the existence of a relatively compact set $K \subset \ell_{2}$ and $\phi: \ell_{2} \longrightarrow X$ such that $A \subset \phi(K)$. Put $x_{n}=\phi\left(e_{n}\right)$ and and $B:=2-\operatorname{co}\left(x_{n}\right)$. To prove that $A$ is relatively compact in $X_{B}$, let us consider the quotient map $Q: \ell_{2} \longrightarrow \ell_{2} / \operatorname{Ker} \phi$ and the operator $\widehat{\phi}: \ell_{2} / \operatorname{Ker} \phi \longrightarrow X$ defined so that $\widehat{\phi}\left(Q\left(\beta_{n}\right)\right)=\phi\left(\beta_{n}\right)$ for every $\left(\beta_{n}\right) \in \ell_{2}$. Then, the following diagram is conmutative:

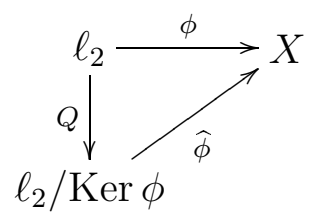

On the other side, it is not difficult to see that the operator $I: \ell_{2} / \operatorname{Ker} \phi \longrightarrow$ $X_{B}$ defined by $I\left(\left[\left(\alpha_{n}\right)\right]\right)=\sum_{n} \alpha_{n} x_{n}$ is an isomorphism between Banach spaces satisfying $\widehat{\phi}=j_{B} \circ I$ :

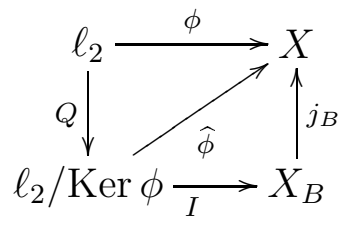

Now, since $j_{B}(A)=A \subset \phi(K)$, it is clear that $\widehat{\phi}\left(I^{-1}(A)\right) \subset \widehat{\phi}(Q(K))$. From the injectivity of $\widehat{\phi}$, it follows that $A \subset I(Q(K))$. 
Conversely, assume that $A \subset X$ verifies (1) and (2). If $\phi$ is the operator induced by the sequence $\left(x_{n}\right)$, then the isomorphism $I: \ell_{2} / \operatorname{Ker} \phi \longrightarrow X_{B}$ defined as above enables to see $X_{B}$ as a Hilbert space. According to [22, Theorem 10.8], $j_{B}^{*}$ is 2-summing and, since $A$ is relatively compact in $X_{B}$, $A=j_{B}(A)$ is relatively 2 -compact in $X$ [8, Theorem 3.14].

As an application, we show a relatively compact set in $c_{0}$ inside of the 2-convex hull of $\left(e_{k}\right)$ but failing to be relatively 2-compact (here, $\left(e_{k}\right)$ denotes the unit vector basis of $c_{0}$ ).

Example 3.2. For each $n \in \mathbb{N}$, put $\left.x_{n}=\left(\frac{1}{\sqrt{n}}, . ..\right), \frac{1}{\sqrt{n}}, 0 \ldots\right) \in c_{0}$ and consider $A=\left\{x_{n}: n \in \mathbb{N}\right\} \subset B:=2$-co $\left(e_{k}\right)$. Then $A$ is relatively compact; in fact,

$$
\lim _{n}\left\|x_{n}\right\|_{\infty}=0
$$

In order to see that $A$ is not relatively $\rho_{B}$-compact, we first prove that $\rho_{B}\left(x_{n}\right)=1$ for all $n \in \mathbb{N}$. By contradiction, assume that there exists $n \in \mathbb{N}$ so that $\rho_{B}\left(x_{n}\right)<1$ and choose $t \in\left[\rho_{B}\left(x_{n}\right), 1\right)$ such that $x_{n} \in t B$. Then

$$
x_{n}=\sum_{n} t \alpha_{k} e_{k}
$$

for a fixed $\left(\alpha_{k}\right)_{k} \in B_{\ell_{2}}$. Thus $\left\langle x^{*}, x_{n}\right\rangle=\sum_{n} t \alpha_{k}\left\langle x^{*}, e_{k}\right\rangle$ for all $x^{*} \in \ell_{1}$. In particular,

$$
\begin{array}{cc}
t \alpha_{k}=\frac{1}{\sqrt{n}} & \text { if } k \leq n \\
t \alpha_{k}=0 & \text { if } k>n .
\end{array}
$$

From this

$$
1 \geq \sum_{k} \alpha_{k}^{2}=\frac{1}{t^{2}},
$$

which is a contradiction to $t<1$. Now, if $A$ is relatively $\rho_{B}$-compact, then there exists a subsequence $\left(x_{k(n)}\right)$ of $\left(x_{n}\right) \rho_{B}$-convergent to $x \neq 0$. Since $j_{B}$ is continuous, $\left(x_{k(n)}\right)$ is $\|\cdot\|_{\infty}$-convergent to $x \neq 0$, a contradiction to (3).

In the previous section, we have also showed that $L_{1}(\mu)$ fails to be in $\mathcal{C}_{p}$ if $p \geq 1$. Anyway, a criterion of 1-compactness in $L_{1}(\mu)$ can be deduced using the characterization of nuclear operators into $L_{1}(\mu)$ due to Grothendieck (see [10, p. 258]):

Proposition 3.2. A bounded subset $A$ of $L_{1}(\mu)$ is relatively 1-compact if and only if

1. $A$ is order bounded, i.e., there exist $g \in L_{1}(\mu)$ such that $|f| \leq g \mu$ almost everywhere for each $f \in A$, and 
2. $A$ is equimeasurable, i.e., given $\varepsilon>0$, there is a measurable set $\Omega_{\varepsilon}$ such that $\mu\left(\Omega \backslash \Omega_{\varepsilon}\right)<\varepsilon$ and $\left\{f \chi_{\Omega_{\varepsilon}}: f \in A\right\}$ is relatively compact in $L_{\infty}(\mu)$.

Proof. If $A \subset L_{1}(\mu)$ is relatively 1-compact, then $U_{A}^{*}$ is nuclear. According to [10, Theorem VIII.3.7], $U_{A}$ is itself nuclear and this leads up to conclude that $A \subset U_{A}\left(B_{\ell_{1}(A)}\right)$ is order bounded and equimeasurable [10, p. 258]. Conversely, let us see that $U_{A}^{*}$ is nuclear whenever $A$ is order bounded and equimeasurable in $L_{1}(\mu)$. For if, notice that $U_{A}\left(B_{\ell_{1}(A)}\right) \subset \operatorname{co}(A)$ is also order bounded and equimeasurable (here, co $(A)$ denotes the closed absolutely convex hull of $A$ ). Then, $U_{A}$ is nuclear, as well as $U_{A}^{*}$.

Since operators from any $\mathcal{L}_{\infty}$-space to any space with cotype 2 are 2summing [9, Theorem 11.14], we can reproduce the proof of Lemma 3.1 to obtain 2-compact sets in $\mathcal{L}_{1}$-spaces.

Proposition 3.3. Let $X$ be a $\mathcal{L}_{1}$-space. Then $A \subset X$ is relatively 2-compact if and only if there exist a relatively compact set $K \subset \ell_{2}$ and an operator $\phi: \ell_{2} \longrightarrow X$ such that $A \subset \phi(K)$.

We finish with some results concerning to the equality $\mathcal{L}\left(Y, \ell_{q}\right)=\mathcal{K}_{p}\left(Y, \ell_{q}\right)$. The following is a consequence of the equality $\mathcal{K}_{p}\left(Y, \ell_{1}\right)=\Pi_{p}^{d}\left(Y, \ell_{1}\right)$ and $[9$, Theorem 11.14].

Proposition 3.4. Let $Y$ be a Banach space such that $Y^{*}$ has cotype $s \geq 2$. We have:

1. If $s=2$, then $\mathcal{L}\left(Y, \ell_{1}\right)=\mathcal{K}_{2}\left(Y, \ell_{1}\right)$.

2. If $s>2$, then $\mathcal{L}\left(Y, \ell_{1}\right)=\mathcal{K}_{p}\left(Y, \ell_{1}\right)$ for every $p>s$.

Corollary 3.3. Let $p \geq 2$. We have:

1. $\mathcal{L}\left(\ell_{r}, \ell_{1}\right)=\mathcal{K}_{2}\left(\ell_{r}, \ell_{1}\right)$ for every $r \geq 2$.

2. If $p>2, \mathcal{L}\left(\ell_{r}, \ell_{1}\right)=\mathcal{K}_{p}\left(\ell_{r}, \ell_{1}\right)$ for every $r>p^{\prime}$.

Remark 3.4. Notice that $\mathcal{L}\left(\ell_{r}, \ell_{1}\right) \neq \mathcal{K}_{2}\left(\ell_{r}, \ell_{1}\right)$ whenever $r<2$. For if, consider an operator $T \in \mathcal{L}\left(c_{0}, \ell_{r^{\prime}}\right)$ failing to be $r^{\prime}$-summing [13, Theorem 7]. Thus, $T^{*} \notin \Pi_{2}^{d}\left(\ell_{r}, \ell_{1}\right)=\mathcal{K}_{2}\left(\ell_{r}, \ell_{1}\right)$. If $p>2$, the same argument can be used to explain that $\mathcal{L}\left(\ell_{r}, \ell_{1}\right) \neq \mathcal{K}_{p}\left(\ell_{r}, \ell_{1}\right)$ whenever $r \leq p^{\prime}$.

If $p<2$, the equality $\mathcal{L}\left(Y, \ell_{1}\right)=\mathcal{K}_{p}\left(Y, \ell_{1}\right)$ implies that $Y$ is finite dimensional. Indeed, if $\mathcal{L}\left(Y, \ell_{1}\right)=\Pi_{p}^{d}\left(Y, \ell_{1}\right)$ holds, it follows that the identity map on $Y^{*}$ is $(p, 1)$-summing, a contradiction to [9, Theorem 10.5].

Now we make clear that, if the rank space is $\ell_{q}$ with $q>1$, then, for each $p \geq 1$, there are bounded operators failing to be $p$-compact.

Proposition 3.5. Let $p \geq 1$ and $q>1$. If $\mathcal{L}\left(Y, \ell_{q}\right)=\mathcal{K}_{p}\left(Y, \ell_{q}\right)$ then $Y$ is finite dimensional. 
Proof. Since $\ell_{q} \in \mathcal{C}_{p}$, then $\mathcal{L}\left(Y, \ell_{q}\right)=\Pi_{p}^{d}\left(Y, \ell_{q}\right)$. According to [15, Theorem 1.3], $\mathcal{L}\left(\ell_{q^{\prime}}, Y^{*}\right)=\Pi_{p}\left(\ell_{q^{\prime}}, Y^{*}\right)$. This implies that $Y^{*}$ must be finite dimensional ([15, p. 22]).

Remark 3.5. The proof of Lemma 3.1 essentially works because $\mathcal{L}\left(\ell_{2}, X\right)=$ $\Pi_{1}^{d}\left(\ell_{2}, X\right)$ if $X$ is a $\mathcal{L}_{\infty}$-space. If $q>1$, the above result reveals that $\mathcal{L}\left(\ell_{2}, \ell_{q}\right) \neq \mathcal{K}_{p}\left(\ell_{2}, \ell_{q}\right)=\Pi_{p}^{d}\left(\ell_{2}, \ell_{q}\right)$. Thus, the procedure used to prove Lemma 3.1 and Proposition 3.3 is not useful to obtain characterizations of $p$-compact sets in $\ell_{q}(q>1)$.

\section{References}

[1] R. Aharoni and P. Saphar, On the reflexivity of the space $\Pi_{p}(E, F)$ of p-absolutely summing operators, $1 \leq p<\infty$, Bull. London Math. Soc., 25 (1993), 362 - 368.

[2] R.M. Aron, M. Maestre and P. Rueda, p-Compact holomorphic mappings, Rev. R. Acad. Cienc. Exactas Fis. Nat. Ser. A Math., 104 (2010), $353-364$.

[3] J.L. Arregui and O. Blasco, $(p, q)$-Summing sequences, J. Math. Anal. Appl., 274 (2002), 812 - 827.

[4] S. Aywa and J.H. Fourie, On summing multipliers and applications, J. Math. Anal. Appl., 253 (2001), 166 - 186.

[5] J. Bourgain and O. Reinov, On the approximation properties for the space $H^{\infty}$, Math. Nachr., 122 (1985), 19 - 27.

[6] Y.S. Choi and J.M. Kim, The dual space of $\left(\mathcal{L}(X, Y), \tau_{p}\right)$ and the $p$ approximation property, J. Funct. Anal., 259 (2010), 2437 - 2454.

[7] J.S. Cohen, Absolutely $p$-summing, p-nuclear operators and their conjugates, Math. Ann., 201 (1973), 177 - 200.

[8] J.M. Delgado, C. Piñeiro and E. Serrano, Operators whose adjoints are quasi p-nuclear, Studia Math, 197 (2010), 291 - 304.

[9] J. Diestel, H. Jarchow and A. Tonge, Absolutely summing operators, Cambridge Univ. Press, Cambridge, 1995.

[10] J. Diestel and J.J. Uhl, Vector measures, Math. Surveys Monogr. 15, Amer. Math. Soc., Providence, RI, 1977.

[11] A. Grothendieck, Produits tensoriels topologiques et espaces nucléaires, Memoirs Amer. Math. Soc., 16, 1955. 
[12] R. Khalil, On some Banach space sequences, Bull. Austral. Math. Soc., 25 (1982), 231 - 241.

[13] S. Kwapien, On a theorem of L. Schwartz and its applications to absolutely summing operators, Studia Math., 38 (1970), 193 - 201.

[14] S. Lassalle and P. Turco, On $p$-compact mappings and the $p$ approximation property, J. Math. Anal. Appl., 389 2012, 1204 - 1221.

[15] J.S. Morrell and J.R. Retherford, p-Trivial Banach spaces, Studia Math., 43 (1972), 1 - 25.

[16] A. Persson, On some properties of $p$-nuclear and $p$-integral operators, Studia Math., 33 (1969), 213 - 222.

[17] A. Persson and A. Pietsch, $p$-Nukleare und p-integrale Abbildungen in Banachräumen, Studia Math., 33 (1969), 19 - 62.

[18] A. Pietsch, Operator Ideals, North-Holland Publishing Company, Amsterdam-New York-Oxford, 1980.

[19] C. Piñeiro and J.M. Delgado, $p$-Convergent sequences and Banach spaces in which $p$-compact sets are $q$-compact, Proc. Amer. Math. Soc., 139 (2011), 957 - 967.

[20] O.I. Reinov, A survey of some results in connection with Grothendieck approximation property, Math. Nachr., 119 (1984), 257 - 264.

[21] D.P. Sinha and A.K. Karn, Compact operators whose adjoints factor through subspaces of $\ell_{p}$, Studia Math., 150 (2002), 17 - 33.

[22] N. Tomczak-Jaegermann, Banach-Mazur Distances and FiniteDimensional Operator Ideals, Pitman Monographs and Surveys in Pure and Applied Mathematics, New York, 1989.

\section{Received: November 11, 2013}

